\title{
Scale up of laboratory scale to industrial scale biogas plants
}

\author{
Alexandra Kowalczyk ${ }^{1, *}$, Sebastian Schwede ${ }^{1}$, Mandy Gerber ${ }^{1}$, Roland Span ${ }^{1}$ \\ ${ }^{1}$ Ruhr-University Bochum, Institute of Thermo and Fluid Dynamics, Bochum, Germany \\ * Corresponding author. Tel: +49 23432 26409, Fax: +49 23432 14163, E-mail: a.kowalczyk@thermo.rub.de
}

\begin{abstract}
Industrial biogas plants often do not operate in their optimum. To investigate limits of anaerobic digestion processes experiments are necessary. Economically it would be feasible to perform these tests at laboratory scale, if the tests could be transferred to industrial scale. This work presents a preliminary study, in which two different scales of laboratory digesters are compared and reproducibility of tests is investigated.

Therefore, three identical glass digesters with a liquid volume of up to 22 liter and a steel-digester with a liquid volume of 390 liter were used. All digesters were started up with digestate from an industrial biogas plant, were fed with cow manure and corn cob mix, and were operated at the same process parameters like temperature, organic loading rate and retention time. Gas volumes were measured continuously. Twice a day the composition of the biogas was analyzed. Dry matter and volatile solids were quantified once a week.

The presented data show a good reproducibility between biogas plants of the same scale. The transferability to a bigger scale is in an acceptable range, but depending on the organic loading rate the deviation between the different scales varies.
\end{abstract}

Keywords: biogas plant, laboratory scale, comparison, reproducibility, transferability

\section{Introduction}

Sources for renewable energy become more and more important. Among the renewable energies biogas has the advantage that it is a not fluctuating source. When being used in the existing pipeline infrastructure, it can even be stored to compensate for fluctuations in consumption. Storage for short periods is possible directly in most plants. Biogas can be gained in an anaerobic digestion process from different organic substances, e.g. from energy crops, agricultural waste or municipal organic waste.

Industrial biogas plants often do not operate in their optimum. To reach a higher efficiency for biogas plants it is necessary to know the limits for the anaerobic digestion process. These limits have to be identified in experiments. Economically it is not feasible to perform these tests on an industrial scale biogas plant since they involve the risk of long lasting production curtailments. Therefore, experiments at laboratory scale are the most common way to study the anaerobic digestion process. To use the results of laboratory scale experiments, it is essential to know whether the results are transferable to industrial scale and whether the experiments are reproducible.

To answer these questions it is necessary to use exactly the same inoculums for all digesters used in one test series and the same substances for feeding, because the anaerobic digestion process is a strongly dynamical process and depends very much on the used material. Furthermore, all digesters investigated should be operated with the same process parameters such as temperature, organic loading rate (OLR), hydraulic retention time and liquid volume for studies on reproducibility. For transferability studies only the liquid volume is allowed to vary. Transferability and reproducibility were investigated by Brunn et al. [1] with a non identical OLR for the different scales. Gallert et al. [2] operated their digesters with different OLR and hydraulic retention times. This work presents results of a pre study in which two different scales of laboratory digesters are compared keeping all process parameters as equal as possible. Furthermore, a reproducibility test was carried out with identical digesters. In coming tests a laboratory scale digester will be compared with an industrial scale biogas plant. 


\section{Methodology}

To prove the reproducibility of experiments on the anaerobic digestion process, three identical continuous (22 liter laboratory) digesters were used. The transferability of experimental results to different scales was investigated comparing the 22 liter digesters to a 390 liter continuous digester.

\subsection{Experimental setup}

One of the three identical digesters with a liquid volume of 22 liter is shown in Fig. 1. The double glass shell of the digester is used for heating and gives the possibility to observe mixing and liquid level. Mixing is realized by a central stirring system with three mixing elements. These are propeller mixers with three blades for every mixing element. At the bottom a ball valve allows to discharge the digester or to take samples. For feeding a ball valve connected with the lid is used. From the top of the digester a gas-pipe leads to a 5 liter gas sampling bag. The gas sampling bag is necessary, to avoid low pressure by draining during feeding or taking samples. From the gas sampling bag a heated pipe leads to a drum type gasmeter (TG 05; Ritter). The gasmeter has a PT-100 thermometer and a manometer to be able to calculate the standard volume flow.
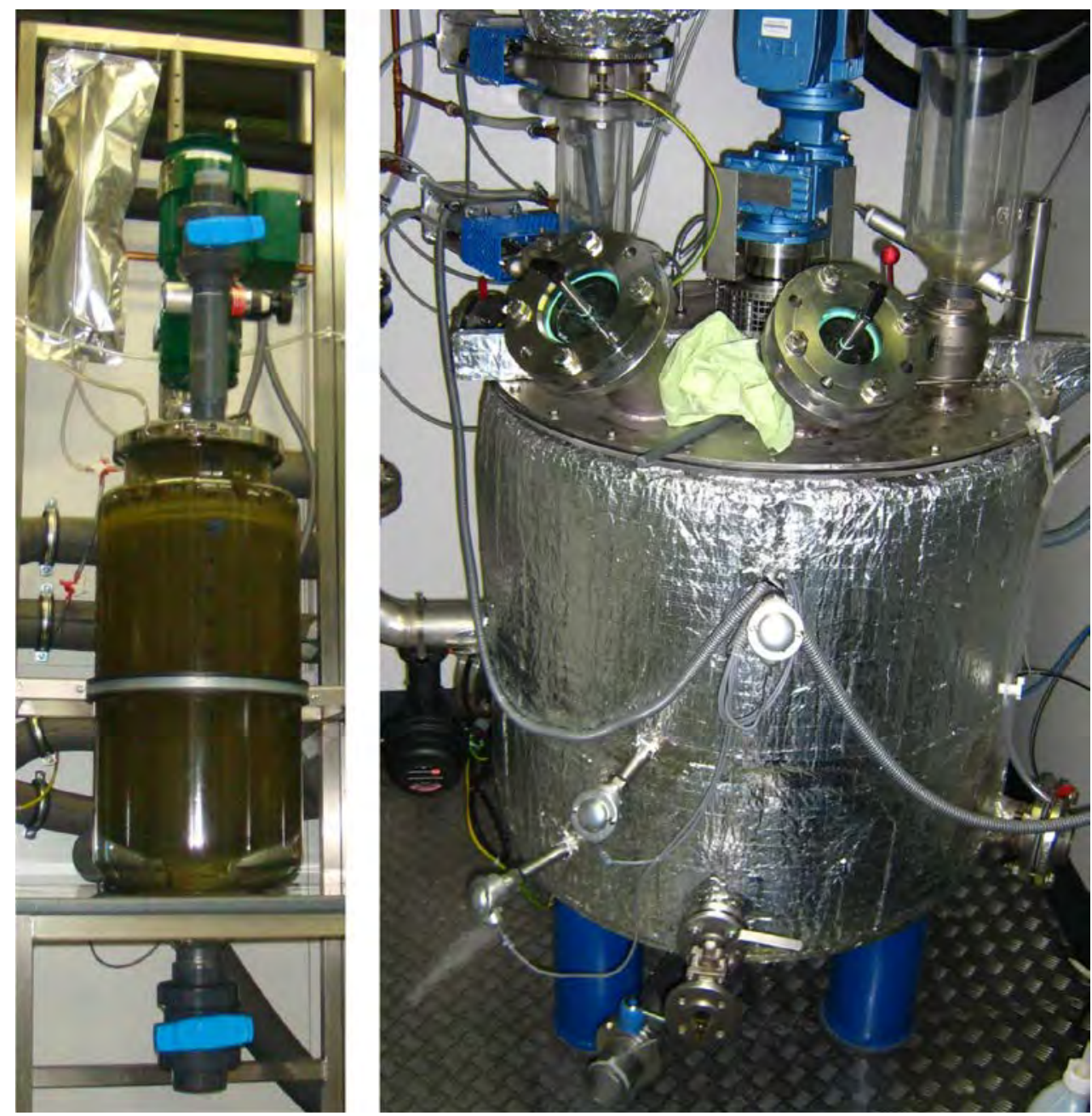

Fig. 1. Left: One of the three continuous 22 l digesters; Right: continuous 390 l digester.

Two identical gas chromatographs (GC) (Focus GC, Thermo Electron Corporation, Axel Semrau) are used to analyze the gas composition. One is equipped with a thermal conductivity 
detector (Thermo Fisher Scientific, Axel Semrau) with a micropacked column (ShinCarbon ST 100/120, Restek) for analyzing methane $\left(\mathrm{CH}_{4}\right)$, carbon dioxide $\left(\mathrm{CO}_{2}\right)$, oxygen $\left(\mathrm{O}_{2}\right)$ and nitrogen $\left(\mathrm{N}_{2}\right)$. The other GC is connected to a mass spectrometer (DSQ II, Thermo Electron Corporation, Axel Semrau) with a capillary column (GS-GasPro, J\&W Scientific Products) for hydrogen sulfide $\left(\mathrm{H}_{2} \mathrm{~S}\right)$. Furthermore a variable over pressure function is connected to the top. To determine the temperature in the digester a PT-100 thermometer is used.

The biogas plant with a liquid volume of 390 liter is shown in Fig. 1. It is made of stainless steel. The geometry of the digester is a scale-down of an industrial plant with $3000 \mathrm{~m}^{3}$ liquid volume. The gas flow is much higher in the 390 liter digester. Therefore, no problems with low pressure occur during feeding or taking samples and a gas sampling bag is not needed. A constant liquid level is held due to a siphon which connects the digester with a storage tank. The digester is mixed by a central stirring system with three mixing elements. These are pitched blade impellers with two blades. This means, that they differ in their geometry compared to the ones of the 22 liter digesters. Temperature is measured on three levels. The digester is equipped with the same metrology as the smaller digesters. The measured quantities and their uncertainties are summarized in Table 1.

Table 1. Measured quantities and their uncertainties.

\begin{tabular}{cccccccc}
\hline$T$ & $p$ & $\dot{V}$ & $\mathrm{CH}_{4}$ & $\mathrm{CO}_{2}$ & $\mathrm{O}_{2}$ & $\mathrm{~N}_{2}$ & $\mathrm{H}_{2} \mathrm{~S}$ \\
$(\mathrm{mK})$ & $(\mathrm{mbar})$ & $(\%)$ & $(\mathrm{mol}-\%)$ & $(\mathrm{mol}-\%)$ & $(\mathrm{mol}-\%)$ & $(\mathrm{mol}-\%)$ & $(\mathrm{ppm})$ \\
\hline $\pm 31,0$ & $\pm 1,5$ & $\pm 0,2$ & $\pm 4,0$ & $\pm 3,4$ & $\pm 0,2$ & $\pm 0,3$ & $\pm 615,3$ \\
\hline
\end{tabular}

\subsection{Experimental performance}

All four digesters were filled with digestate from an industrial biogas plant (fed with maize silage (MS), corn cob mix (CCM) and cow manure (CM)) at the beginning. During the test period of 40 days the digesters were run at the same temperature level of ca. $38^{\circ} \mathrm{C}$. The test period of 40 days was considered to be sufficient, after the digesters showed a good reproducibility and transferability even with changing organic loading rates (OLR). Once a day they were fed with the same mixture of CCM and CM, which was mashed with digestate. CM was chosen because of its good buffer capacity. CCM and CM are, compared e.g. to MS, very homogeneous and well suited for reproducibility and transferability tests. The chosen feeding rates ensured the same OLR for all digesters. The OLR is calculated according to

$O L R=\frac{\dot{m}_{V S}}{V_{l i q}}$

where $\dot{m}_{V S}$ is the input mass flow of volatile solids (VS) and $V_{\text {liq }}$ the liquid volume of the respective digester. The OLR was increased from 0.20 to $2.54 \mathrm{kgvs}_{\mathrm{vs}} \mathrm{d}^{-1}$ during the test period. The steps are shown in Fig. 2. 


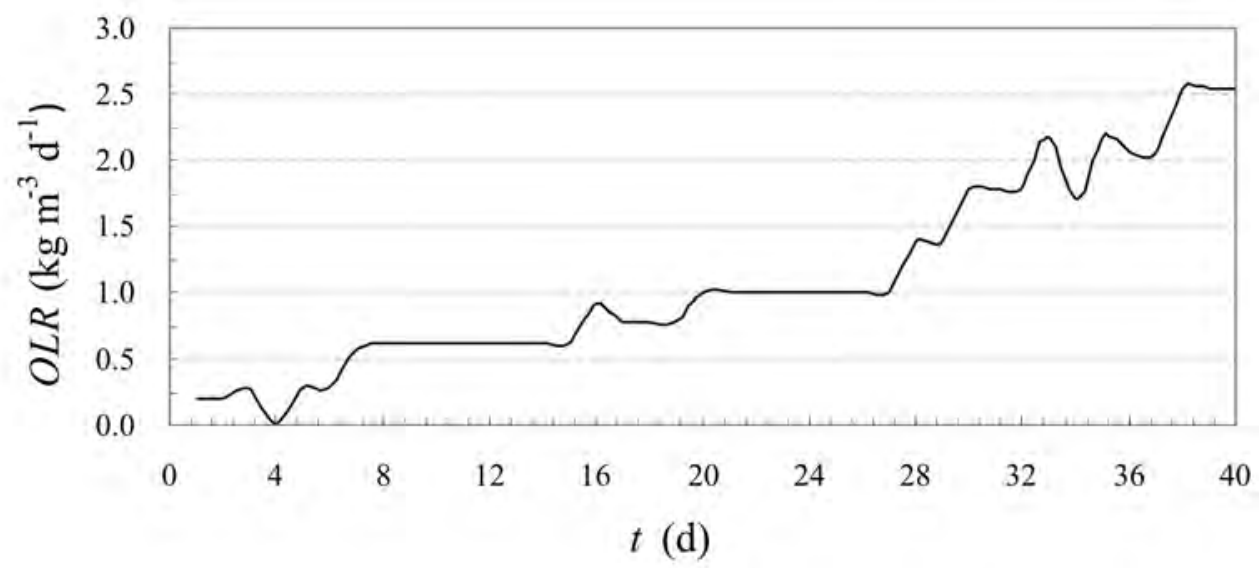

Fig. 2. OLR over test period for all digesters.

Before and after feeding gas samples were taken for gas quality analysis. During the whole test period the gas flow was measured continuously and the digesters have been stirred without any interruptions. Six times during the test period of 40 days dry matter (DM) and volatile solids (VS) where determined according to DIN 12879 [3] and DIN 12880 [4], respectively.

\section{Results}

During the test period the four plants showed a good agreement with respect to the analyzed parameters. Fig. 3 and Fig. 4 give an overview of the performance for two different days. The diagrams show the standard volume flow reduced by the liquid volume of the digester over one day. The reduction is necessary, because otherwise it would not be possible to compare the 22 liter digesters to the 390 liter digester. The drop in Fig. 3 and at 05:00 and 08:00 o'clock in Fig. 4 result from sampling for gas analysis. The fluctuations between 10:00 and 15:00 o'clock are caused by feeding and taking samples for gas analysis.

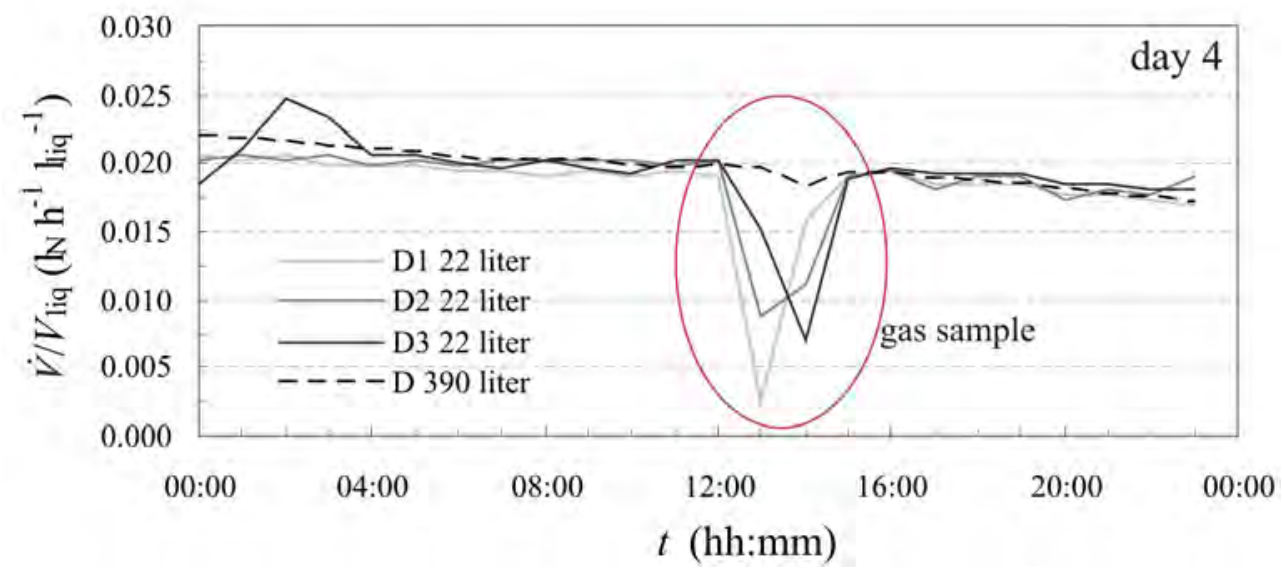

Fig. 3. Volume flow referring to the filling volume for fourth day (D: Digester). 


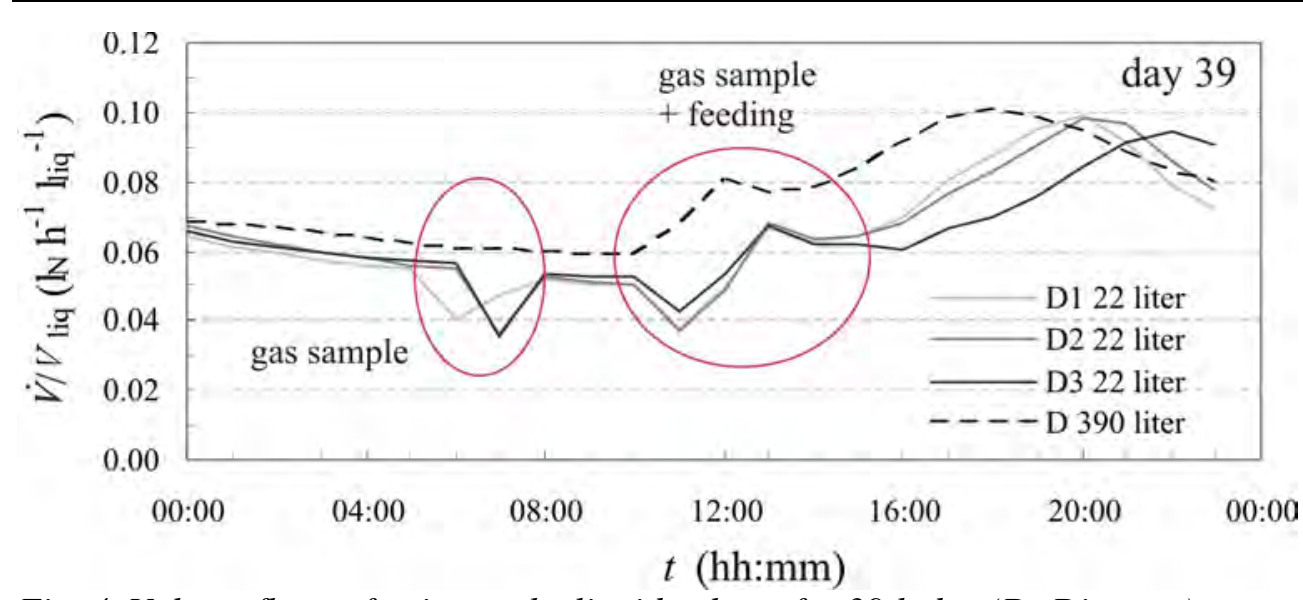

Fig. 4. Volume flow referring to the liquid volume for 39th day (D: Digester).

Fig. 3 shows a nearly identical shape of the curve with very similar results for all four digesters. In Fig. 4 the curve of the $390 \mathrm{l}$ digester deviates from the ones of the $22 \mathrm{l}$ digesters. The trends at the two different scales are comparable at any time, for derivates. In this example the curve of the third 22 liter digester is displaced on the time-line, but shows the same trends.

Fig. 5 shows the daily average of the absolute standard deviation for the 22 liter digesters, as well as the daily average of the absolute deviation between the 390 liter digester and the average of the three 22 liter digesters. To calculate the deviation the values during feeding or taking gas samples, as described in Fig. 3 and Fig. 4, and outliers were excluded. On the second axis the average of the daily gas volume flow to the liquid volume of the digesters is shown. Here the outliers are excluded as well.

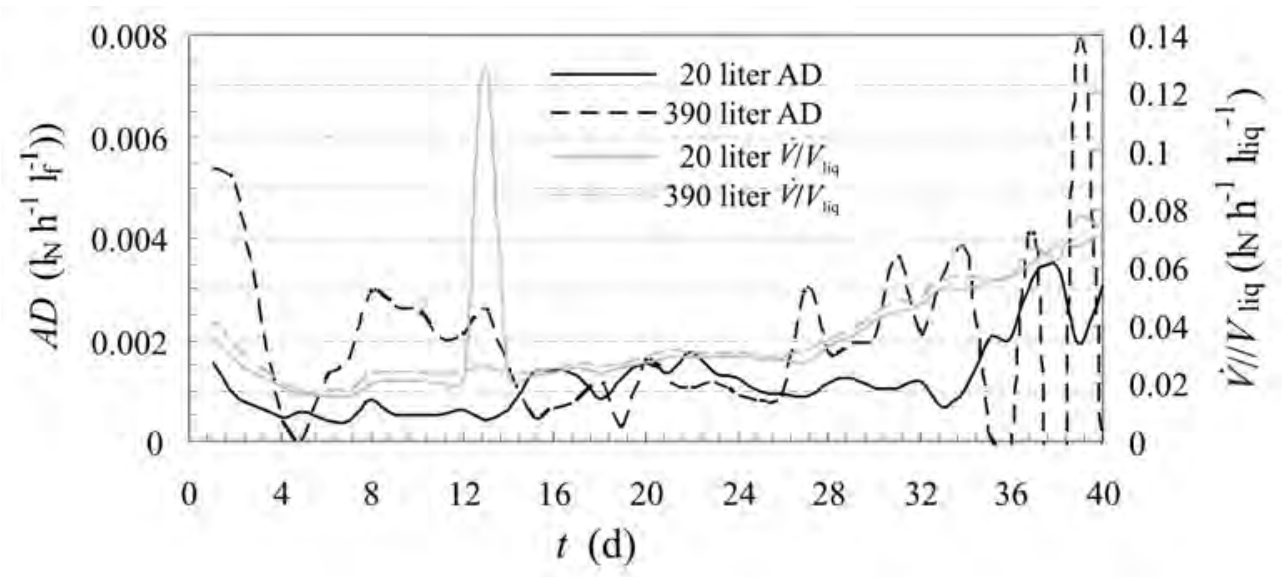

Fig. 5. Daily average of the absolute standard deviation (AD) for the three 22 liter digesters and daily average of the absolute deviation $(A D)$ between 390 liter digester. Also the average of the three 22 liter digesters and the daily average of the gas volume flow reduced by the liquid volume for 22 liter and 390 liter digesters.

Fig. 5 shows a good reproducibility of the three $22 \mathrm{l}$ digesters among each other. The relative standard deviation is between 1.42 and $5.96 \%$ (excluded one outliner with $21.58 \%$ on day 13) for the 22 liter digesters among each other. Comparing the average of the $22 \mathrm{l}$ digesters to the $390 \mathrm{l}$ digester the relative deviation is between 0.68 and $18.07 \%$. This and the shape of the curves for the gas volume flow in Fig. 5 for both digester scales are an evidence for a possible transferability of experimental results in different scales. 


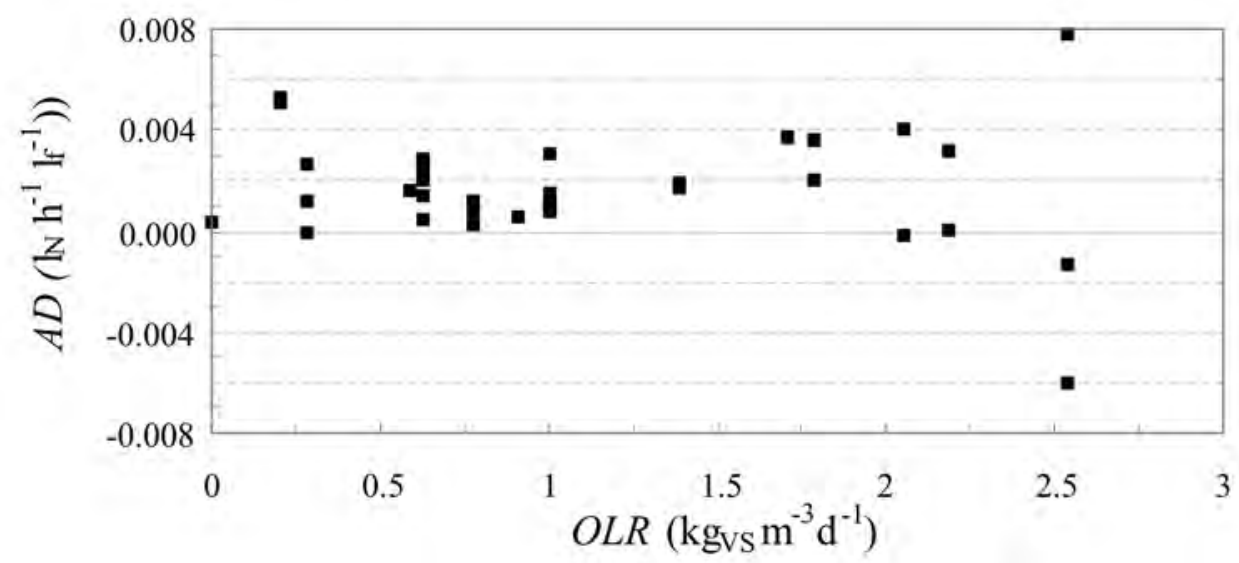

Fig. 6. Absolute deviation $(A D)$ of the 390 liter digester to the average of the 22 liter digesters over OLR.

Fig. 6 shows the absolute deviation of 390 liter to 22 liter digesters (referring to gas volume flow to liquid volume) over OLR. For each OLR, expect $2.53 \mathrm{~kg}_{\mathrm{vs}} \mathrm{m}^{-3} \mathrm{~d}^{-1}$, the deviation is between 0.0002 and $0.0053 \mathrm{l}_{\mathrm{N}} \mathrm{h}^{-1} \mathrm{l}_{\mathrm{f}}^{-1}$. For an OLR of $2.53 \mathrm{kgvs}^{-3} \mathrm{~d}^{-1}$ the absolute deviation is between -0.0061 and $0.0078 \mathrm{l}_{\mathrm{N}} \mathrm{h}^{-1} \mathrm{l}_{\mathrm{f}}^{-1}$.

To compare the biogas quality, Fig. 7 shows the plot of the measured methane concentration. Expect for the curve of the first 22 liter plant at days 14-18 and a single peak of the $390 \mathrm{l}$ digester at the end of the test period, all curves show a good agreement. The difference between the two scales till day 6 results from the gas sampling bags used for the 22 liter digesters, which contain air at start up.

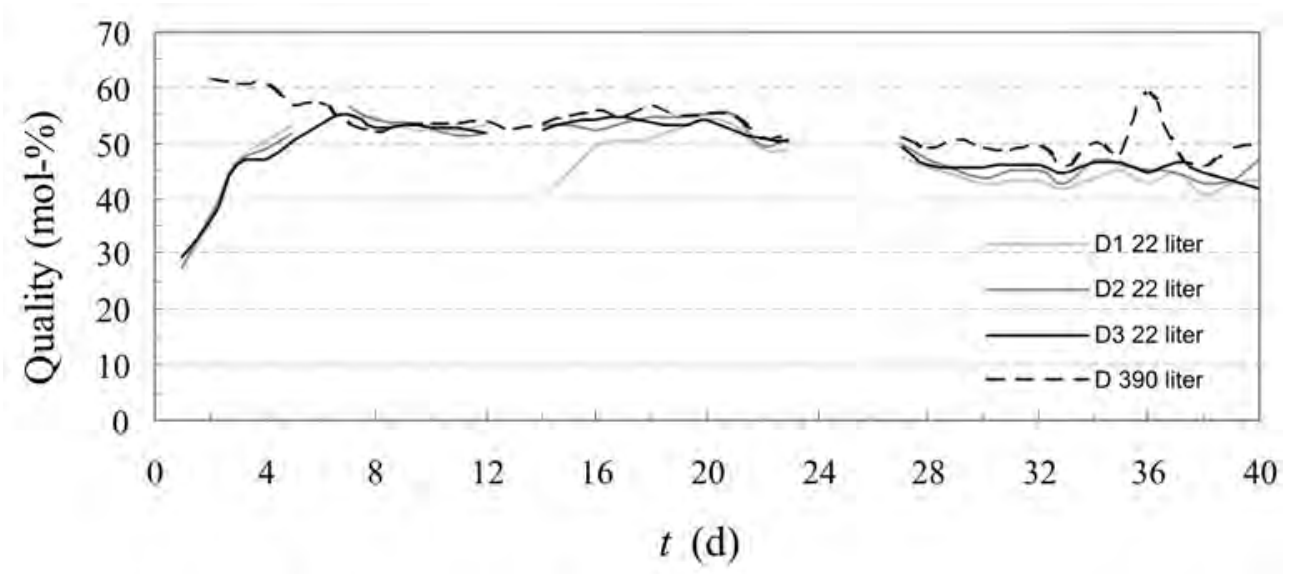

Fig. 7. Methane-rate over test period.

Table 2 shows results for DM and VS determined during the test. Day 0 represents the starting point, at which all digesters have been filled with the same inoculum. After the first week the $\mathrm{DM}$ in the $390 \mathrm{l}$ digester decreases more than in the smaller digesters. This indicates a higher conversion of the substrates to biogas. This is also shown in Fig. 5, where the production rate of the 390 liter digester is always above the one of the 22 liter digesters. 
Table 2. Dry matter (DM) and organic dry matter (VS) of all digesters through the test period.

\begin{tabular}{|c|c|c|c|c|c|c|c|c|}
\hline$t$ & (d) & & 0 & 7 & 16 & 23 & 29 & 36 \\
\hline \multirow{4}{*}{$D M$} & \multirow{4}{*}{$\left(\% \mathrm{FM}^{*}\right)$} & BP1 22 l & 8,37 & 8,10 & 7,85 & 7,61 & 7,47 & 7,63 \\
\hline & & BP2 221 & 8,37 & 8,30 & 7,67 & 7,42 & 7,67 & 7,81 \\
\hline & & BP3 22 l & 8,37 & 8,33 & 7,55 & 7,37 & 7,48 & 7,61 \\
\hline & & BP 3901 & 8,37 & 7,72 & 7,38 & 7,11 & 7,11 & 7,57 \\
\hline \multirow{4}{*}{ VS } & \multirow{4}{*}{$\left(\% \mathrm{FM}^{*}\right)$} & BP1 221 & 6,47 & 6,00 & 5,65 & 5,50 & 5,64 & 5,76 \\
\hline & & BP2 221 & 6,47 & 5,83 & 5,56 & 5,53 & 5,47 & 5,88 \\
\hline & & BP3 22 l & 6,47 & 6,00 & 5,65 & 5,39 & 5,45 & 5,73 \\
\hline & & BP 3901 & 6,47 & 5,92 & 5,63 & 5,43 & 5,43 & 5,76 \\
\hline
\end{tabular}

* fresh mass

\section{Conclusion and Outlook}

The presented results show a high degree of reproducibility at equal experimental conditions. During the test period the daily relative standard deviation of the three 22 liter digesters is between 1.42 and $5.96 \%$. Reasons for this deviation are the heterogeneity of the fed substrates and small differences in liquid volume and digester temperature.

The relative deviation of the $390 \mathrm{l}$ digester to the three 22 liter digesters is between -6.92 and $18.07 \%$ with an average of $6.33 \%$. This is not caused by the OLR, which was varied during the test. The reasons for the deviation have to be searched in the same causes as the ones for the reproducibility. Additional reasons are the differences in geometry, in materials used for the digesters and in mixing.

Good correspondence between a laboratory scale digester and a full scale digester was observed by Gallert et al. [2]. This is a good indicator, that experiments in laboratory scale can be transferred to industrial scale. Aivasidis and Wandrey [5] concluded, that it is possible to scale up anaerobic digesters and that experiments in laboratory and pilot scale can provide data to design an industrial scale digester.

Also Brunn et al [1] figured out a good reproducibility for digesters of the same scale, but not for transferability. The industrial scale digester produces $36 \%$ more gas, compared to the used laboratory scale digester. As explained by Brunn et al. this causes in different feeding schedules and substrates (substrates for the laboratory digester were taken once a week). This shows the importance of similar process parameters for tests relating to reproducibility and transferability.

The next step in studying the transferability of experimental results should be a comparison between a digester at laboratory scale with one at industrial scale. All process parameters have to be chosen as identical as possible, as the presented results underline the importance of synchronicity of all process parameters. Currently the industrial biogas plant is being equipped with the necessary measure devices.

\section{Acknowledgements}

We thank RWE Innogy GmbH for supporting the presented work. 


\section{References}

[1] L. Brunn, C. Dornack, B. Bilitewski, Application of laboratory scale experiments to industrial scale in case of anaerobic waste treatment, Fresenius Environmental Bulletin 18 (2), 2009, pp. 196-203

[2] G. Gallert, A. Henning, J. Winter, Scale-up of anaerobic digestion of the biowaste fraction from domestic wastes, Water Research 37, 2003, pp. 1433-1441

[3] DIN EN 12879, Characterization of sludges - Determination of the loss on ignition of dry mass, 2001

[4] DIN EN 12880, Characterization of sludges - Determination of dry residue and water content, 2001

[5] A. Aivasidis, C. Wandrey, Development and Scaleup of a high-rate biogas process for treatment of organically polluted effluents, Annals of the New York Academy of Sciences 589, 1990, pp 599-615 